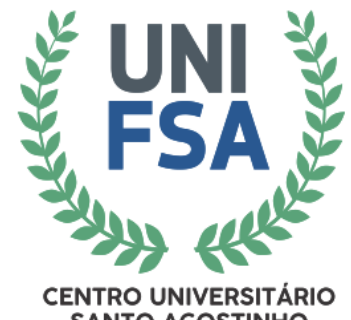

SANTO ACOSTINHO
CENTRO UNIVERSITÁRIO SANTO ACOSTINHO

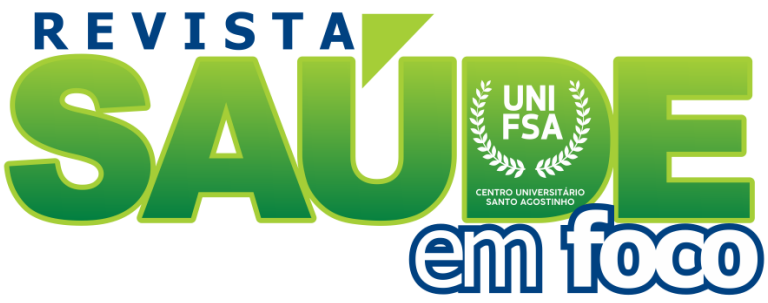

www4.fsanet.com.br/revista

Revista Saúde em Foco, Teresina, v. 7, n. 3, art. 6, p. 76-100, set./dez. 2020

ISSN Eletrônico: 2358-7946

http://dx.doi.org/10.12819/rsf.2020.7.3.6

\title{
A Atuação do Farmacêutico na Prevenção às Intoxicações Exógenas por Medicamentos Psicotrópicos
}

The Performance of the Pharmacist in the Prevention of Exogenous Intoxications by Psychotropic Drugs

Carla Cshunderlick Graduação no curso de Farmácia da Universidade Guarulhos

E-mail: carlacshunderlick@gmail.com

Cláudia Raquel Zamberlam Doutora em Ciências pela UNIFESP Docente do curso de Farmácia da Universidade Guarulhos E-mail: crzamberlam@gmail.com
Endereço: Carla Cshunderlick Endereço: Pça. Tereza Cristina, 88 - Centro - Guarulhos SP; CEP: 07023-070, Brasil.

Endereço: Cláudia Raquel Zamberlam

Endereço: Pça. Tereza Cristina, 88 - Centro - Guarulhos SP; CEP: 07023-070, Brasil.
Editor-Chefe: Dr. Tonny Kerley de Alencar Rodrigues

Artigo recebido em 05/12/2020. Última versão recebida em 07/07/2021. Aprovado em 08/07/2021.

Avaliado pelo sistema Triple Review: a) Desk Review pelo Editor-Chefe; e b) Double Blind Review (avaliação cega por dois avaliadores da área).

Revisão: Gramatical, Normativa e de Formatação 


\title{
RESUMO
}

Os medicamentos psicotrópicos agem no sistema nervoso central e são usados no tratamento de transtornos mentais ou distúrbios psiquiátricos. Eles causam tolerância e dependência e, por esta razão, devem ter dispensação controlada e exclusiva dos farmacêuticos se enquadrando na Portaria $\mathrm{n}^{\circ}$ 344/98 do Ministério da Saúde do Brasil. No entanto, sua utilização de maneira abusiva é cada vez mais comum, podendo levar a intoxicações exógenas e a óbitos. Como principal objetivo deste trabalho, buscou-se salientar a importância do farmacêutico na orientação para a prevenção da reincidência de intoxicações por estes tipos de medicamentos. $\mathrm{O}$ método utilizado na pesquisa incluiu uma revisão bibliográfica através da reunião de informações das principais publicações de 2013 a 2020, coletadas de diversos bancos de dados virtuais, além de dados epidemiológicos disponíveis no Sistema Nacional de Informações Tóxico-Farmacológicas (SINITOX) de 2015 a 2017 e diversos livros, leis, resoluções e portarias a respeito do assunto. Tal análise trouxe considerações finais importantes acerca do papel fundamental do farmacêutico como interceptor nestas situações, uma vez que é o último profissional da saúde a intervir antes que o usuário tome seu medicamento. No ato da dispensação dos medicamentos, após uma cuidadosa avaliação da prescrição médica, o farmacêutico deverá orientar o paciente, enfatizando o cumprimento da posologia, os males da interação com outros medicamentos e bebidas alcoólicas, o reconhecimento de reações adversas potenciais e os problemas que a sobredose pode ocasionar. Dessa forma, buscará proporcionar o uso racional e seguro dos medicamentos e, por conseguinte, garantir a efetividade e segurança terapêutica.

Palavras-chaves: Assistência Farmacêutica. Toxicologia. Psicotrópicos. Saúde Mental. Sistema Nervoso Central.

\begin{abstract}
Psychotropics medications act on the central nervous system and are used in the treatment of mental disorders or psychiatric disorders. Since they cause tolerance and dependence, they must have controlled and exclusive dispensation of pharmacists complying into Ordinance No. 344/98 of the Brazilian Ministry of Health. However, it's use in an abusive manner is increasingly common, which can lead to exogenous intoxications and deaths. The main objective of this monograph was to emphasize the importance of the pharmacist in guiding the prevention the recurrence of intoxications by these types of medications. The method used in the research included a literature review carried out by gathering information on the main publications collected in the virtual databases from 2013 to 2020, in addiction to epidemiological data available at the National Poisoning Information System (SINITOX) from 2015 to 2017 and several books, laws, resolutions and ordinances on the subject. This analysis brought important final considerations about the key role of the pharmacist as an interceptor in these situations, since it's the last health professional to intervene before the user takes his medication. In the act of dispensing medications, after a careful evaluation of the medical prescription, the pharmacist should guide the patient emphasizing the fulfilment of the dosage, the harm of interaction with other drugs and alcohol, the recognition of potential adverse reactions and the problems that overdose may cause. In this way, it will seek to provide the rational and safe use of medicines, and will ensure effectiveness and therapeutic safety.
\end{abstract}


Keywords: Pharmaceutical Care. Toxicology. Psychotropics. Mental Health. Central Nervous System.

\section{INTRODUÇÃO}

Os psicotrópicos (psique $=$ mente e tropico $=$ alteração), psicofármacos ou fármacos psicoativos são medicamentos que agem no sistema nervoso central, indicados para o tratamento de transtornos mentais ou distúrbios psiquiátricos, e que pertencem à Portaria $\mathrm{n}^{\circ}$ 344, de 12 de maio de 1998 (BRASIL, 1998a), da Secretaria de Vigilância em Saúde (SVS/MS), conhecidos como remédios de "tarja preta", são substâncias que podem causar tolerância, dependência física, química ou psíquica (COUTO et al., 2015).

São eles os antipsicóticos, ansiolíticos, anticonvulsivantes, antidepressivos, hipnóticos, estabilizadores de humor e psicoestimulantes, que têm grande risco de gerarem intoxicações se utilizados de maneira abusiva ou inadequada, afetando as funções mentais e emocionais, podendo levar a danos, ou ao óbito dos usuários, sendo frequentemente utilizados para autoextermínio (CARVALHO et al., 2017).

Os casos e óbitos devido a intoxicações por medicamentos crescem diariamente, seja por falta de informação, seja por abuso intencional. Como dizia Paracelsus (1493 - 1541), “todas as substâncias são venenos, não existe nada que não seja veneno. Somente a dose correta diferencia o veneno do remédio", ou seja, a magnitude da exposição (dose) pode torná-los tóxicos ou fatais. Portanto, é essencial o estudo das intoxicações por estes tipos de drogas, para dimensionar o problema, subsidiar discussão sobre uso e acesso desses medicamentos e nortear práticas clínicas para redução de danos e prevenção de novas intoxicações (SANTOS; LEGAY; LOVISI, 2013).

Estes medicamentos teoricamente só podem ser dispensados pelos farmacêuticos mediante a apresentação de duas vias de receituário médico de controle especial, sendo a primeira via retida pelo farmacêutico e a segunda, devolvida ao paciente, e uma via de notificação de receita (NR) numerada na cor azul ou amarela. Mas há algumas exceções, pois alguns farmacêuticos meramente os vendem, sem se importar com os males que seu uso sem prescrição podem causar aos usuários, o que é uma das causas dos casos e óbitos por esses tipos de fármacos, simplesmente pela negligência e falta de orientação destes profissionais (BRASIL, 1998a).

Outro fator que pode desencadear este problema é a prescrição médica feita por médicos psiquiatras, neurologistas e até mesmo clínicos gerais, após um diagnóstico 
impreciso ou equivocado da doença, dado que atualmente não há testes que de fato a comprove, de modo a prescreverem medicamentos desnecessários (GONÇALVES et al., 2017).

Contribuir com a literatura sobre a importância do farmacêutico na orientação para o uso seguro de medicamentos psicotrópicos. Abordar a Toxicologia dos medicamentos psicotrópicos; Promover o uso racional e seguro de medicamentos, e garantir sua eficiência terapêutica; Associar o uso abusivo de psicofármacos à falta de orientação farmacêutica.

\section{METODOLOGIA}

Com base nas bibliografias publicadas, disponíveis em artigos e revistas científicas, teses e dissertações, através do acesso aos bancos de dados virtuais Scielo, BDTD, LILACS, BVS, PubMed, PubChem, Google Acadêmico e SINITOX. Além da consulta a diversos livros, leis, resoluções e portarias a respeito do assunto, foi feita a revisão bibliográfica para o desenvolvimento deste trabalho. Sendo as principais publicações datadas entre 2013 a 2020, os dados epidemiológicos mais recentes, coletados do SINITOX dos anos de 2015 a 2017, a soma e o percentual médio dos dados, calculados utilizando-se o Microsoft Office Excel 2013.

\section{REFERENCIAL TEÓRICO}

\subsection{Intoxicação exógena por medicamentos}

Intoxicação exógena (IE) é a resposta à interação de um ou mais agentes tóxicos com o organismo, devido ao rompimento do equilíbrio orgânico pelo toxicante, que produz alterações fisiológicas e bioquímicas, ocasionado o surgimento de um estado patológico como resposta (SANTOS; LEGAY; LOVISI, 2013; CARVALHO et al., 2017).

As intoxicações exógenas por medicamentos, sejam eles ingeridos, inalados, injetados, sejam pelo contato com a pele, olhos ou membranas mucosas, podem ocorrer em razão da automedicação, erros de prescrição e de administração, exposição acidental, tentativas de suicídio e aborto, homicídio, abuso ou interação medicamentosa (IM) (GONÇALVES et al., 2017).

As IEs só ocorrem quando o agente tóxico atinge a corrente sanguínea, manifestando seus efeitos nocivos, através de sinais e sintomas (estado patológico), que revelam o 
desequilíbrio orgânico decorrente da ingestão de medicamentos em doses acima das terapêuticas, podendo causar danos ou levar ao óbito, dependo da sua gravidade intoxicações agudas ou crônicas (CARVALHO et al., 2017; BALEN et al., 2017; SILVA; SILVEIRA, 2019).

Na capital de São Paulo, segundo a Associação Brasileira de Centros de Informação e Assistência Toxicológica e Toxicologistas Clínicos (ABRACIT) existem três Centros de Informação e Assistência Toxicológica (CIATs), responsáveis por registrar o número de casos e óbitos de intoxicações por diversos fatores (ABRACIT, 2012):

- $\quad$ Centro de Controle de Intoxicações - CCI de São Paulo;

- Instituto Butantan - Hospital Vital Brazil;

- $\quad$ Centro de Assistência Toxicológica - CEATOX do Instituto da Criança (Hospital das Clínicas/USP).

\subsubsection{Fases da intoxicação}

Fase de exposição: corresponde ao contato do toxicante com o organismo através das vias de administração (CETESB, [21--]);

Fase toxicocinética: é caracterizada pela chegada do toxicante ao sítio de ação e divide-se em processos pelos quais permite-se a movimentação do AT no organismo:

- Absorção - corresponde à passagem do AT do local de entrada para a circulação sistêmica, seja no trato gastrointestinal, pulmonar ou cutâneo;

- Distribuição - consiste na fase em que a fração livre do composto deve atravessar as membranas celulares para alcançar o órgão alvo, podendo durante o processo ligar-se ao tecido formando depósitos de armazenamento;

- Biotransformação ou metabolismo - é responsável por modificações estruturais no fármaco, alterando sua atividade farmacológica e velocidade de excreção, através de reações de fase I - oxidação, redução e hidrólise, e de fase II - glicoronidação, sulfatação, acetilação, metilação, conjugação com glurationa e conjugação com aminoácidos, tornando os metabólitos mais hidrossolúveis ou polares;

- Excreção ou eliminação - ocorre através de secreções como a biliar, sudorípara, lacrimal, gástrica, salivar e láctea, e através de excreções como a urina, por meio de três mecanismos: filtração glomerular, secreção tubular e difusão tubular passiva; fezes; catarro e pelo ar inalado por difusão simples (MARQUES, 2018).

Fase toxicodinâmica: corresponde às ações do toxicante sobre o organismo, assim que ele ou seu metabólito alcançam o tecido-alvo, causando-lhe alterações moleculares, 
bioquímicas ou fisiológicas, que serão responsáveis por seu efeito tóxico (danos) (PEREIRA; FRANCO-BERNARDES; DORTA, 2018).

Fase clínica: corresponde à manifestação clínica dos efeitos resultantes da ação tóxica, através de sinais e sintomas (PARANÁ, [21--]).

\subsubsection{Dados epidemiológicos de intoxicações por medicamentos}

O Sistema Nacional de Informações Tóxico-Farmacológicas (SINITOX) é o órgão responsável pela publicação de dados estatísticos anuais referentes a casos e óbitos por intoxicação registrados pelos Centros de Informação e Assistência Toxicológica (CIATs), que têm critérios de faixa etária, sexo, circunstância, zona de ocorrência e evolução (SANTOS; LEGAY; LOVISI, 2013).

Os dados coletados correspondem aos anos de 2015 a 2017, visto que estes consistem nas últimas informações publicadas, com enfoque no número de casos e óbitos por medicamentos, segundo faixa etária e sexo (SINITOX, 2015 - 2017).

As crianças de um a quatro anos são as vítimas mais frequentes das intoxicações acidentais ocasionadas por medicamentos, respondendo com $24,2 \%$ dos casos registrados no Brasil (Tabela 1). O que ocorre geralmente em função do armazenamento inadequado destes fármacos por seus familiares, de modo a ficarem a uma altura acessível e que, por curiosidade e desconhecimento dos riscos que podem causar, acabam ingerindo-os. A segunda faixa etária mais afetada é constituída pelos jovens adultos, de 20 a 29 anos, com 13,7\% dos casos registrados (NUNES et al., 2017).

Tabela 1 - Casos de intoxicação por medicamentos, segundo faixa etária. Brasil, 2015 à 2017

\begin{tabular}{c|c|c|c|c} 
Faixa Etária & $\mathbf{2 0 1 5}$ & $\mathbf{2 0 1 6}$ & $\mathbf{2 0 1 7}$ & Total \\
\hline$<\mathbf{1}$ & 1103 & 1192 & 551 & 2846 \\
$\mathbf{0 1 - 0 4}$ & 7831 & 8206 & 3730 & 19767 \\
\hline $\mathbf{0 5 - 0 9}$ & 2033 & 2123 & 1207 & 5363 \\
\hline $\mathbf{1 0 - 1 4}$ & 1329 & 1511 & 1071 & 3911 \\
\hline $\mathbf{1 5 - 1 9}$ & 2303 & 2622 & 2248 & 7173 \\
\hline $\mathbf{2 0 - 2 9}$ & 3909 & 3977 & 3335 & 9746 \\
\hline $\mathbf{3 0 - 3 9}$ & 3483 & 3612 & 2651 & 6808 \\
\hline $\mathbf{4 0 - 4 9}$ & 2431 & 2498 & 1879 & 4549 \\
\hline $\mathbf{5 0 - 5 9}$ & 1671 & 1779 & 1099 & 2636 \\
\hline $\mathbf{6 0 - 6 9}$ & 1079 & 1107 & 450 & \\
\hline
\end{tabular}




\begin{tabular}{c|c|c|c|c}
\hline $\mathbf{7 0 - 7 9}$ & 783 & 735 & 244 & 1762 \\
\hline $\mathbf{8 0} \mathbf{e}+$ & 375 & 421 & 135 & 931 \\
\hline Ignorado & 448 & 2528 & 2037 & 5013 \\
\hline Total & 28878 & 32311 & 20637 & 81726
\end{tabular}

Fonte: SINITOX/FIOCRUZ, 2015 - 2017 (Adaptado)

Quanto ao sexo, as mulheres são as maiores vítimas (Tabela 2), correspondendo a $61 \%$ dos casos e dos óbitos registrados e isso deve-se ao fato de aderirem a farmacoterapias com maior frequência e geralmente não usarem métodos tão agressivos em tentativas de suicídio quanto os homens, que em sua maioria submetem-se a enforcamentos ou ao uso de armas brancas ou de fogo nestas situações, sendo assim mais suscetíveis às intoxicações medicamentosas (NUNES et al., 2017).

Tabela 2 - Casos e óbitos de intoxicação por medicamentos, segundo sexo. Brasil,

\begin{tabular}{l|l|l|l|l|l|l}
\multicolumn{2}{l}{} & \multicolumn{2}{c}{$\mathbf{2 0 1 5}$ à 2017} & $\mathbf{2 0 1 6}$ & $\mathbf{2 0 1 7}$ & \multicolumn{1}{l}{} \\
\cline { 2 - 8 } & Casos & Óbitos & Casos & Óbitos & Casos & Óbitos \\
\cline { 2 - 8 } Masculino & 10501 & 16 & 11389 & 11 & 6150 & 14 \\
\hline Feminino & 18176 & 28 & 19199 & 18 & 12566 & 19 \\
\hline Ignorado & 101 & 18 & 1723 & 86 & 1921 & 17
\end{tabular}

Fonte: SINITOX/FIOCRUZ, 2015 - 2017 (Adaptado)

Os óbitos mais comuns são entre pessoas das faixas etárias de 30 a 49 anos (Tabela 3) (SINITOX, 2015 - 2017).

Tabela 3 - Óbitos de intoxicação por medicamentos, segundo faixa etária. Brasil, 2015 à 2017

\begin{tabular}{c|c|c|c|c} 
Faixa Etária & $\mathbf{2 0 1 5}$ & $\mathbf{2 0 1 6}$ & $\mathbf{2 0 1 7}$ & Total \\
\hline$<\mathbf{1}$ & 1 & 2 & - & 3 \\
\hline $\mathbf{0 1 - 0 4}$ & 2 & - & - & 2 \\
\hline $\mathbf{0 5 - 0 9}$ & - & - & 1 & 1 \\
\hline $\mathbf{1 0 - 1 4}$ & - & - & 3 & 10 \\
\hline $\mathbf{1 5 - 1 9}$ & 4 & 3 & 5 & 18 \\
\hline $\mathbf{2 0 - 2 9}$ & 9 & 6 & 5 & 21 \\
\hline $\mathbf{3 0 - 3 9}$ & 9 & 11 & 4 & 14 \\
\hline $\mathbf{4 0 - 4 9}$ & 11 & 5 & 1 & 6 \\
\hline $\mathbf{5 0 - 5 9}$ & 5 & 5 & 4 & 5 \\
\hline $\mathbf{6 0 - 6 9}$ & 1 & & & 2 \\
\hline
\end{tabular}




\begin{tabular}{c|c|c|c|c}
\hline $\mathbf{7 0 - 7 9}$ & 2 & 3 & 1 & 6 \\
\hline $\mathbf{8 0} \mathbf{e}+$ & - & - & - & - \\
\hline Ignorado & 18 & 76 & 27 & 121 \\
\hline Total & 62 & 115 & 50 & 227
\end{tabular}

Fonte: SINITOX/FIOCRUZ, 2015 - 2017 (Adaptado)

No Brasil, tem-se o costume de manter estoques domiciliares de medicamentos para automedicação, guarda para uso futuro e sobras de tratamentos anteriores, o que favorece o acesso e consequentemente o risco das intoxicações. Outro fator predisponente é o incorreto descarte de medicamentos vencidos ou sem uso pela população, de modo que seus resíduos potencialmente tóxicos podem contaminar o solo e a água quando descartados no lixo ou na rede de esgoto comum, causando efeitos teratogênicos, mutagênicos e carcinogênicos em humanos e animais (FERREIRA et al., 2017), além do risco de serem utilizados por pessoas que trabalham em lixões, ocasionando envenenamentos e até morte (BRASIL, 2015; SANTOS; OLIVEIRA; SALVI, 2015).

Outro agravante é o uso simultâneo ou consecutivo de cinco ou mais medicamentos por uma única pessoa, denominado polifarmácia ou politerapia, sendo mais comum em idosos e pacientes com doenças crônicas como hipertensão arterial e diabetes (DA SILVA; ÁLVARES, 2019), o que pode causar interações medicamentosas, que consistem nas alterações dos efeitos ou da farmacocinética de um fármaco devido à administração prévia ou coadministração de um segundo fármaco e que em casos de adição, sinergismo e potenciação do efeito terapêutico, resultam frequentemente em intoxicações medicamentosas (BALEN et al., 2017; CARVALHO, 2018).

Além disso temos a publicidade direta ao consumidor, que consiste nas propagandas vinculadas à mídia e mesmo nas farmácias e drogarias. Estas estimulam o uso dos medicamentos de venda livre ou MIPs - medicamentos isentos de prescrição médica - para tratar, geralmente, cefaleia, dismenorreia, flatulência, pirexia, dorsalgia, gripe e resfriado (SANTANA et al., 2018).

Salienta-se que, para solucionar estes problemas, compete às políticas públicas de saúde implementar vigorosamente o sistema de fracionamento de medicamentos, em que apenas se fornece ao paciente a quantidade exata para o tratamento. Quanto ao descarte de medicamentos, realizado muitas vezes de maneira inadequada, há também a necessidade de políticas de incentivo de logística reversa (CONSTANTINO et al, 2020).

Embora exista a Política Nacional de Medicamentos (PNM), criada pela Portaria $\mathrm{n}^{\mathrm{o}}$ 3.916/98, do Ministério da Saúde (GM/MS), que tem como objetivo precípuo garantir a 
necessária segurança, eficácia e qualidade dos medicamentos, a promoção do uso racional e o acesso da população àqueles considerados essenciais, não tem se mostrado tão eficaz na redução de episódios de intoxicação medicamentosa (BRASIL, 1998b).

\subsection{Medicamentos psicotrópicos}

Os medicamentos psicotrópicos, psicoativos ou psicofármacos são substâncias sujeitas a controle especial pela Portaria $n^{\circ} 344 / 98$ (BRASIL, 1998a) da Agência Nacional de Vigilância Sanitária (ANVISA), constantes nas listas "A3", "B1" e "B2", devendo conter frases de alerta na rotulagem das embalagens primárias e secundárias, dentro da faixa preta (BRASIL, 2010), conforme a Figura 1.

Figura 1 - Tarja Preta

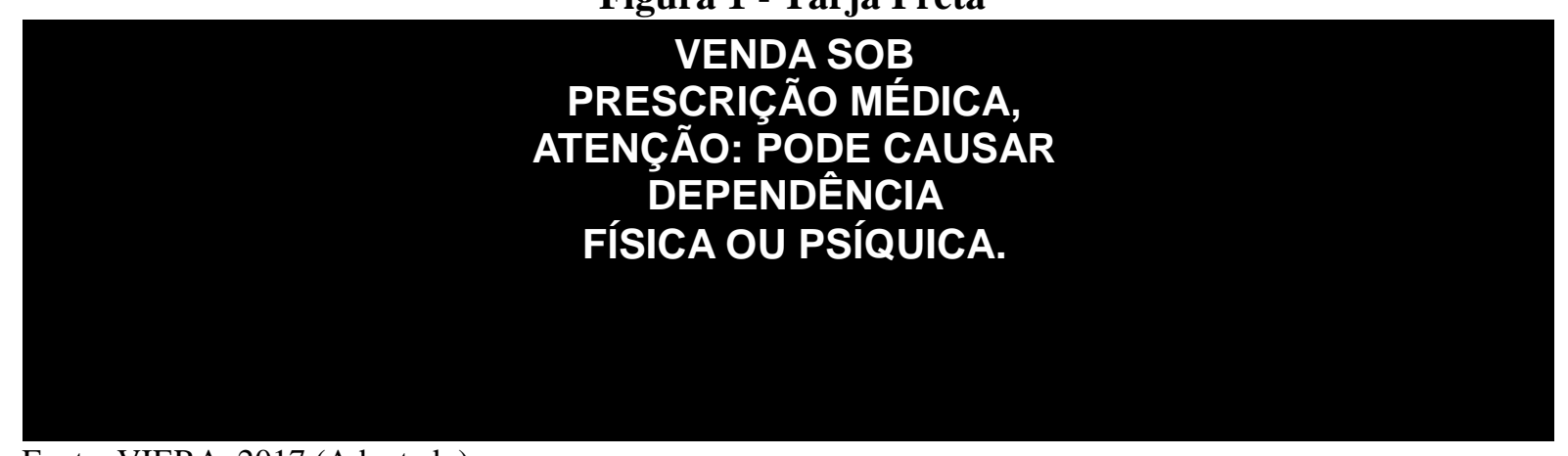

Fonte: VIERA, 2017 (Adaptado).

Sua dispensação é exclusiva do farmacêutico e só deve ocorrer mediante a apresentação de duas vias de receituário médico de controle especial, sendo a $1^{\text {a }}$ via retida na farmácia ou drogaria e a $2^{\mathrm{a}}$ via devolvida ao paciente para orientação sobre o uso correto dos medicamentos prescritos, e uma via de notificação de receita (NR) numerada na cor amarela, para os medicamentos que constam na lista "A3" ou azul, para os que pertencem às listas "B1" e “B2” (CONSTANTINO et al., 2020).

Segundo a Organização Mundial da Saúde (OMS), agem no sistema nervoso central (SNC), através de sua estimulação, perturbação ou depressão, alterando a liberação de neurotransmissores (NT), sendo os principais a norepinefrina (NA) ou noradrenalina (NA), dopamina (DO), histamina, acetilcolina (ACh), serotonina (5-HT) e ácido aminobutírico (GABA), produzindo alterações de comportamento, humor e cognição (BRASIL, 2010), isto é, afetam as funções mentais e emocionais, podendo ser agrupadas conforme seus princípios ativos (COUTO et al., 2015; FRACO; KRIEGER, 2016). 
Entre esses grupos estão: ansiolíticos (calmantes ou tranquilizantes), antipsicóticos (neurolépticos) típicos e atípicos, anticonvulsivantes (antiepilépticos), antidepressivos, estabilizadores de humor (antimaníacos), psicoestimulantes e hipnóticos (sedativos ou soníferos). Utilizados no combate a transtornos mentais, que representam $13 \%$ do total de todas as patologias do mundo e são definidos segundo o Manual Diagnóstico e Estatístico de Transtornos Mentais (DSM), como ansiedade, dependência química, depressão, esquizofrenia, transtorno bipolar, transtorno obsessivo-compulsivo (TOC), transtorno de déficit de atenção com hipertatividade (TDAH), fobia social, angústia, autismo, distúrbios do sono (insônia), epilepsia, convulsões, distúrbios orgânicos como demência ou delirium, transtorno de estresse pós-traumático e desvios de personalidade. Estes fármacos utilizados para tratar estas doenças são modificadores seletivos do SNC e potencialmente perigosos, sendo fundamental a sua associação com a psicoterapia para resultados mais promissores (ROSA; WINOGRAD, 2013; SILVA; SILVEIRA, 2019).

Os medicamentos mais utilizados são os benzodiazepínicos, como o diazepam e barbitúricos, como o tiopental (ansiolíticos e hipnóticos); haloperidol, clorpromazina e levomepromazina (neurolépticos típicos); quetiapina, clozapina, risperidona, olanzapina, sulpirida e amissulprida (neurolépticos atípicos); os antidepressivos, de acordo com a sua classificação em antidepressivos tricíclicos (ADT); inibidores da monoamina oxidase (IMAO), como: fenelzina, tranilcipromina e moclobemida; inibidores seletivos da receptação de serotonina ou 5-HT (ISRS), como: citalopram, escitalopram, fluoxetina, sertralina e paroxetina; e antidepressivos atípicos ou heterocíclicos; fenobarbital, ácido valproico, carbamazepina, fenobartbital, alprazolan, topiramato e fenitoína (anticonvulsivantes); carbonato de lítio (estabilizador de humor); metilfenidato, lindexanfetamina e modafinil (psicoestimulantes); rivastigmina, memantina e donepezil (antidemenciais), metilfenidato (psicoestimulante) (SANTOS; OLIVEIRA; SALVI, 2015; NOGUEIRA, 2017; PANDE; AMARANTE; BAPTISTA, 2018).

Salienta-se que o uso de psicofármacos, embora minimize os sintomas e as angústias característicos do transtorno mental, não os cessam por completo e de modo definitivo, isto é, não proporcionam a cura, mantendo crises de ansiedade, sintomas psicóticos e até mesmo ideação suicida, mas auxiliam na reintegração do indivíduo ao meio familiar e social (CARVALHO, 2018).

Sua dosagem terapêutica tem como objetivo promover efeitos principais, que são os benéficos ao tratamento, no entanto podem ocasionar reações adversas (RAM) e efeitos secundários ou colaterais, sendo mais comuns, boca seca, insônia ou hipersonia, amnésia, 
diminuição da disposição física e do desejo sexual, disfunção sexual, aumento do peso corporal, pensamentos de morte, ideias e atos suicidas, confusão mental e efeitos extrapiramidais. Além de causar tolerância e dependência química, física ou psíquica, a suspensão de seu uso abruptamente pode causar síndromes ou crises de abstinência, seus efeitos tóxicos podem ser potencializados pela ingestão de bebidas alcoólicas e outros psicofármacos, em consequência de interações medicamentosas, podendo levar ao estado de coma como resultado da diminuição da atividade cerebral (SANTOS; OLIVEIRA; SALVI, 2015; CARVALHO, 2018).

Com propósito de administrá-los, foi criado o Sistema Nacional de Gerenciamento de Produtos Controlados (SNGPC), para monitorar as movimentações de entrada (compras e transferências) e saída (vendas, transformações, transferências e perdas) de medicamentos comercializados em farmácias e drogarias privadas do país, em especial os medicamentos sujeitos à Portaria no 344/98 (PANDE; AMARANTE; BAPTISTA, 2018).

Assim como a maior parte dos medicamentos, especialmente aqueles com ação no SNC, são lipossolúveis, atravessando com mais facilidade a barreira hematoencefálica (BHE), o que dificulta sua eliminação, sendo necessário biotransformá-los, transformando-os em hidrossolúveis, aumentando assim o risco de intoxicações em função do maior tempo de meia-vida ( $\mathrm{T}$ 1/2) e possíveis mecanismos de bioativação, responsáveis por tornar a toxicidade mais alta do que a do precursor (MARQUES, 2018).

\subsubsection{Intoxicação por psicofármacos}

As intoxicações exógenas (IE) por psicofármacos, geralmente classificadas em moderadas ou graves, ocorrem com maior frequência pelo uso de ansiolíticos e antidepressivos, principalmente pelo uso de benzodiazepínicos como o clonazepam e diazepam, associados à tentativas de suicídio, sendo o consumo destes medicamentos predominante em mulheres, pois têm maior prevalência dos transtornos mentais comuns (TMC), caracterizados por sintomas depressivos, estado de ansiedade, insônia, fadiga, queixas somáticas inespecíficas, esquecimento, irritabilidade e dificuldades de memória e de concentração, também em virtude de violência sexual ou doméstica, gravidez indesejada e por preocuparem-se mais em cuidar da saúde, frequentam com maior frequência os serviços médicos, sendo consequentemente as maiores vítimas (SANTOS; OLIVEIRA; SALVI, 2015; CARVALHO, 2018). 
Motivações como esta também são frequentes em idosos que não conseguem lidar com a depressão severa, perdas de filhos e cônjuges, privação de afeto ou abandono, presença de doenças graves e degenerativas e são usuários de múltiplos medicamentos, pois muitos apresentam doenças crônicas e os utilizam para controlar ou prevenir os sintomas causados por essas patologias (ROCHA; WERLANG, 2013; CARVALHO, 2018).

As crianças são também as principais vítimas destas intoxicações, mesmo que acidentalmente, sendo em sua maioria fatais, devido ao seu metabolismo, acabam por absorver mais rapidamente os agentes tóxicos dos psicofármacos e, consequentemente, muitas das vezes não há tempo hábil para salvá-las (SILVA; SILVEIRA, 2019).

Diversos são os fatores que podem desencadeá-las como a introdução de novos psicofármacos no mercado farmacêutico, a autoadministração, a baixa adesão ao tratamento, a sobredosagem inadvertida, o uso descomedido ou irregular destes medicamentos e também o consumo concomitante com bebidas alcoólicas e drogas ilícitas (CARVALHO, 2018).

Atualmente não há testes científicos que comprovem a presença ou ausência de desordens mentais, ou mesmo o suposto desequilíbrio químico apontado por muitos médicos psiquiatras, neurologistas e mesmo clínicos gerais, fator que influencia nas prescrições destes medicamentos, como recurso terapêutico único para tratá-las, sem que haja um diagnóstico preciso de transtornos mentais ou psicológicos, processo conhecido como medicamentalização, assim qualquer desvio, variação ou dificuldade vira anormalidade, e quase todo quadro sintomático persistente pode ser relacionado a algum tipo de transtorno mental, ocasionando as intoxicações (ROSA; WINOGRAD, 2013; FILARDI, 2015; FILARDI; MENDONÇA; RAMALHO-DE-OLIVEIRA, 2019).

Muitas vezes o sofrimento psíquico ou emocional relacionado à vida cotidiana como a tristeza normal, diagnosticada como depressão, é combatida com antidepressivos; a ansiedade, com tranquilizantes; a desatenção e a dispersão, com ritalina, e mesmo o estresse, a tensão, a irritação, medo e cansaço são tratados com esses medicamentos, sem que sejam detectados problemas mentais como distúrbios e/ou transtornos (ROSA; WINOGRAD, 2013; FILARDI, 2015; BALEN et al., 2017; PANDE; AMARANTE; BAPTISTA, 2018; FILARDI; MENDONÇA; RAMALHO-DE-OLIVEIRA, 2019).

Destaca-se que não se descarta a utilização dos psicotrópicos, pois em determinadas circunstâncias podem ser fundamentais, o que se discute é a maneira desordenada e exacerbada com a qual são prescritos (SILVA; HERZOG, 2015). 


\subsubsection{Diagnóstico das intoxicações}

De forma geral, o diagnóstico pode ser feito através da análise das manifestações clínicas que afetam o sistema nervoso (sonolência, rebaixamento sensório, reação extrapiramidal) e o gastrointestinal (cefaleia, vômito, dor gástrica e náusea), como efeitos da sobrecarga destes sistemas. Mas é necessário primeiramente questionar o paciente a respeito da história da exposição para obter-se os dados relacionados ao paciente (histórico médico) e o histórico de exposição que inclui qual é e a quantidade do agente tóxico ou substância, quando (horário), onde e o motivo (tentativa de homicídio, suicídio, acidental, abuso de drogas) (SANTOS; OLIVEIRA; SALVI, 2015; CARVALHO, 2018; SÃO PAULO, 2017).

Em seguida, devem ser realizados exames físicos, para verificação das denominadas "síndromes autonômicas" comuns, variáveis que incluem: pressão arterial, pulsação, sudorese excessiva, tamanho da pupila (míose - pupila contraída, e midríase - pupila dilatada) e atividade peristáltica. E, por fim, os exames complementares de rotina - exames laboratoriais, eletrocardiograma (ECG), exames de imagem (raio X e tomografia computadorizada) ou endoscopia digestiva alta, e toxicológicos, a partir de testes de triagem e de confirmação (FERRARI et al., 2013; SANTOS; OLIVEIRA; SALVI, 2015; SILVA; HERZOG, 2015; NOGUEIRA, 2017).

\subsubsection{Tratamentos}

Há diversos tratamentos para intoxicações por psicofármacos, como terapias de suporte, que consistem basicamente em monitorar os sinais vitais, fazer hidratação adequada e realizar exames de rotina, e a descontaminação, que consiste na eliminação do agente tóxico do organismo com o intuito de diminuir a sua absorção, realizada através de descontaminação do trato gastroinstestinal, geralmente, dividida em duas etapas, sendo a primeira a realização da lavagem gástrica, permitindo o esvaziamento gástrico e consequente eliminação da substância tóxica ingerida, e a segunda, a administração de carvão ativado que possui ação de adsorção do agente tóxico, sendo em alguns casos recomendada a entubação endotraqueal pelas vias nasotraqueal ou orotraqueal e, em outros, a êmese. Seguido da observação clínica, indicada para registro da evolução do paciente, estabilidade dos sinais vitais e superação das manifestações clínicas (SÃO PAULO, 2017).

Para alguns tipos de intoxicação por medicamentos psicotrópicos, existem antídotos específicos, substâncias que agem no organismo, atenuando ou neutralizando ações ou efeitos 
de outras substâncias químicas. Como o bicarbonato de sódio utilizado para alcalinização sanguínea em intoxicações por antidepressivos tricíclicos, sendo que em casos graves é administrada a emulsão lipídica intravenosa; o medicamentio flumazenil utilizado em intoxicação por benzodiazepínicos; L-carnitina indicado em caso de níveis séricos de ácido valproico acima de $450 \mathrm{mg} / \mathrm{mL}$; e o medicamento diazepam usado se for necessária a sedação do paciente, como quando apresentam convulsões (OSLON, 2014; CARVALHO, 2018).

\subsection{Assistência Farmacêutica (AF)}

A assistência farmacêutica (AF) é definida como o conjunto de ações e de serviços que visam assegurar a assistência terapêutica integral, a promoção, a proteção e a recuperação da saúde nos estabelecimentos públicos e privados que desempenhem atividades farmacêuticas, tendo o medicamento como insumo essencial e visando ao seu acesso e uso racional. Ressaltase que é responsabilidade do poder público assegurá-la à população, segundo os princípios e diretrizes do Sistema Único de Saúde (SUS), de universalidade, equidade e integralidade (CORADI, 2012; BRASIL, 2014; ARAÚJO; JUNGES, 2015).

A AF tem como foco o medicamento e consiste em um ciclo, conforme a Figura 2, dividido em seis etapas: seleção, programação, aquisição, armazenamento distribuição e dispensação (e prescrição), que objetivam sua conservação contra fatores extrínsecos (luz, umidade e temperatura) que podem afetar sua estabilidade, e o controle de qualidade, visando segurança, eficácia e custo-efetividade, de modo que cada etapa depende da anterior e contribui diretamente para a viabilidade da seguinte (CORADI, 2012).

Estas etapas são ainda classificadas como serviços farmacêuticos técnico-gerenciais (seleção, programação, aquisição, armazenamento, distribuição), que têm por finalidade garantir a disponibilidade adequada de medicamentos, sua qualidade e conservação, e técnicoassistenciais (dispensação, promoção do uso racional de medicamentos, orientação farmacêutica, seguimento farmacoterapêutico), que garantam a efetividade e segurança da terapêutica e sua avaliação, obtenção e difusão de informações sobre medicamentos e sobre saúde na perspectiva da educação em saúde e educação permanente da equipe de saúde (BRASIL, 2009). 


\section{Figura 2 - Ciclo de Assistência Farmacêutica}

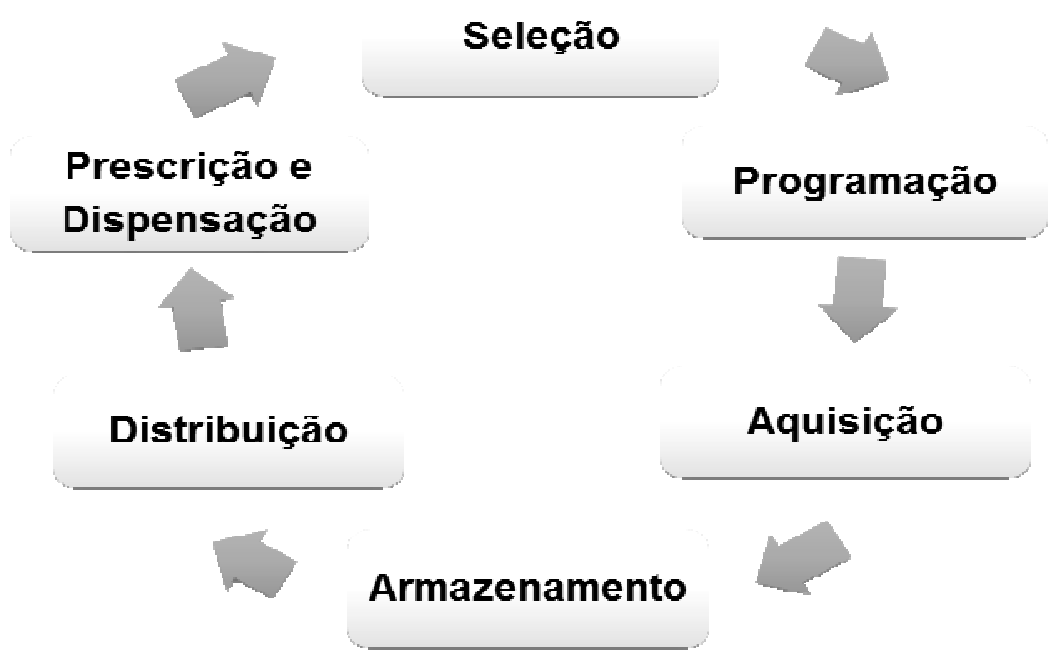

Fonte: CORADI, 2012 (Adaptado).

Seleção é o processo de escolha de medicamentos, baseado em critérios epidemiológicos, técnicos e econômicos; programação consiste em estimar quantidades a serem adquiridas para atendimento a determinada demanda de serviços, por determinado período de tempo; aquisição compreende a compra dos medicamentos; armazenamento envolve um conjunto de atividades para garantir a qualidade físico-química e conservação do medicamento; distribuição compreende suprimentos de medicamentos às unidades de saúde, em quantidade, qualidade e tempo oportuno, e dispensação é a disponibilização do medicamento a um paciente, mediante apresentação de prescrição médica legível e sem rasuras (SILVA; LIMA, 2017; BIZZO et al., 2018; LAGO; ARGOLO; 2019).

$\mathrm{Na}$ etapa de dispensação é fundamental a atuação do profissional farmacêutico que, a partir da prescrição médica, seja em estabelecimentos comerciais (farmácias com e sem manipulação ou drogarias), de assistência ou serviços de saúde (hospitais e unidades básicas de saúde), deve informar e orientar os usuários e seus familiares, a fim de garantir a eficácia e segurança terapêutica e o uso racional de medicamentos (URM), de acordo com a dosagem descrita e o período de tratamento necessário, conforme indicado na Figura 3, por conseguinte não limitando-se apenas em entregar os medicamentos. Outra ação oportuna a este profissional nesta situação é a prescrição farmacêutica, definida conforme a Resolução CFF n ${ }^{\circ}$ 586/13, como:

[...] Ato pelo qual o farmacêutico seleciona e documenta terapias farmacológicas e não farmacológicas, e outras intervenções relativas ao cuidado à saúde do paciente, visando à promoção, proteção e recuperação da saúde, e à prevenção de doenças e de outros problemas de saúde. (COSELHO FEDERAL DE FARMÁCIA, 2013, p.3). 
De modo a assim identificar, reduzir ou até mesmo corrigir riscos associados à farmacoterapia (BRASIL, 2014; ARAÚJO; JUNGES, 2015; ARAÚJO et al., 2017; SANTANA et al., 2018).

Figura 3 - Processo de dispensação de medicamentos na atenção básica Acolhimento ao usuário

Abordar o usuário ou responsável de maneira a apreender suas necessidades

\section{Avaliação da prescrição}

Analisar a adequação da prescrição ao usuário através da análise do receituário e da análise da terapia prescrita.

\section{Separação e preparação do medicamento}

Fornecer medicamentos de qualidade na quantidade necessária ao tratamento conferindo os aspectos quantitativos e qualitativos do medicamento e realizando técnicas adequadas de preparação.

\section{Orientação no fornecimento do medicamento \\ Fornecer as informações necessários ao usuário ou responsável orientando para o processo e os resultados do uso de medicamento.}

\section{Registro de dados e informações gerados}

Abastecer o sistema de controle de estoque e de acompanhamento do usuário registrando o fornecimento dos medicamentose o atendimento prestado.

\section{Dispensação realizada}

Fonte: SARTOR; FREITAS, 2014 (Adaptado).

A última etapa do ciclo de AF deve ser associada à farmacovigilância, definida como “a ciência e atividades relativas à identificação, avaliação, compreensão e prevenção de efeitos adversos ou quaisquer problemas relacionados ao uso de medicamentos", sendo 
incumbido ao farmacêutico notificar através do VigiMed sobre queixas técnicas ou eventos adversos a medicamentos (OMS, 2005; BRASIL, 2014; SANTANA et al., 2018).

Destaca-se que é de suma importância que, para as prescrições médicas, o farmacêutico, o médico e o paciente (ou dispensador, prescritor e usuário) firmem uma relação de coparticipação, em que todos se comprometam a promover o uso racional de medicamentos (ARAUJO, 2016; GRETZLER et al., 2018).

Desenvolvida no contexto da assistência farmacêutica, a atenção farmacêutica (ATF), é considerada como um modelo de prática farmacêutica, em que há a interação direta do farmacêutico com os pacientes, visando à adesão do tratamento farmacológico, uma farmacoterapia racional e a obtenção de resultados definidos e mensuráveis, voltados para a melhoria da qualidade de vida (BRASIL, 2004; SANTANA et al., 2018).

Nesta circunstância, há ainda o acompanhamento ou seguimento farmacoterapêutico (STF), em que o farmacêutico torna-se responsável pelas necessidades do usuário, relacionadas ao medicamento, através da detecção, prevenção e resolução de problemas relacionados a medicamentos (PRM), que seguem os princípios de necessidade, efetividade e segurança, próprios da farmacoterapia, com objetivo de alcançar resultados e melhorar a qualidade de vida do paciente. Este processo compreende diversos métodos, os mais conhecidos são o SOAP (Subjetivo, Objetivo, Avaliação e Plano); o PWDT (Pharmacist's Workup of Drug Therapy), atualmente denominado Pharmacotherapy Workup (PW), e também conhecido como modelo Minnesota; o TOM (Therapeutic Outcomes Monitoring) ou Monitorização de Resultados Terapêuticos; e o Dáder: Método Dáder de Seguimento Farmacoterapêutico (FERREIRA; MELO, 2016).

Atualmente as políticas públicas em saúde mental no Brasil recomendam que portadores de transtornos mentais sejam acompanhados simultaneamente na atenção básica de saúde e em unidades especializadas, sendo as UBS (Unidade Básica de Saúde) e os CAPS (Centro de Atenção Psicossocial) os locais mais procurados e onde o farmacêutico, junto a uma equipe multidisciplinar, poderá atuar colaborando para que o tratamento destes pacientes seja mais efetivo, em atividades tais como: dispensação, orientação farmacêutica, educação em saúde, atendimento farmacêutico, e acompanhamento ou seguimento farmacoterapêutico (ZANELLA; AGUIAR; STORPIRTIS, 2015; ARAUJO, 2017; FERREIRA et al., 2017; BIZZO et al., 2018; COSTA, 2019). 


\section{CONSIDERAÇÕES FINAIS}

Os medicamentos, conforme apontado no SINITOX, são os principais causadores das intoxicações exógenas, desdobradas em quatro fases: exposição, toxicocinética, toxicodinâmica e clínica. Sendo as vítimas mais comuns de internações hospitalares, quanto à faixa etária, crianças de zero a quatro anos, jovens adultos de 20 a 29 anos, e de óbitos, pessoas de 30 a 49 anos, e quanto ao sexo, as mulheres são as mais afetadas, para ambos os parâmetros. Associam-se estes acontecimentos à dosagem acima da terapêutica, seja por fator proposital ou acidental.

Deve-se atenção especial aos medicamentos psicotrópicos - antipsicóticos, ansiolíticos, anticonvulsivantes, antidepressivos, hipnóticos, estabilizadores de humor e psicoestimulantes - que agem seletivamente deprimindo, estimulando ou perturbando o SNC, alterando as funções cognitivas e motoras e são utilizados no tratamento de transtornos mentais ou distúrbios psiquiátricos, sendo de controle especial pela Portaria $\mathrm{n}^{\circ}$ 344/98 (SVS/MS), nas listas “A3", "B1" e "B2", em virtude de causarem tolerância, dependência e crises de abstinência, se houver redução das doses diárias ou descontinuação abrupta da farmacoterapia, além de reações adversas e efeitos colaterais, sendo sua dispensação exclusiva mediante a apresentação obrigatória de duas vias de receituário médico de controle especial e uma via de notificação de receita pelo utente.

As vítimas mais comuns de intoxicações por psicofármacos são mulheres, crianças e idosos por fatores específicos, sobretudo pelo uso de ansiolíticos e antidepressivos. O diagnóstico é feito por meio da investigação minuciosa das manifestações clínicas, histórico médico e de exposição ao agente tóxico, e da realização de exames físicos e complementares, havendo ainda tratamentos e antídotos específicos, que são efetivamente promissores na recuperação.

Inúmeros são os fatores suscitantes destas situações, como a presença de estoques domiciliares de medicamentos; a polifarmácia; as interações medicamentosas; o descarte incorreto de medicamentos; a utilização simultânea com bebidas alcoólicas; a lipossolubilidade dos psicofármacos, dificultando sua excreção; o uso abusivo ou inadequado; a prescrição médica sem diagnóstico preciso de doenças mentais, entre outros.

Para prevenir o crescimento de casos e óbitos envolvendo intoxicações por medicamentos psicoativos, temos os serviços farmacêuticos técnico-assistenciais, em que o farmacêutico, estando em uma posição de autoridade, visto que é o último profissional a 
intervir antes que o usuário tome seu medicamento, poderá na dispensação após uma minuciosa avaliação da prescrição médica na busca por eventuais erros de medicação, orientar o paciente enfatizando o cumprimento da posologia, os males da interação com outros medicamentos e bebidas alcoólicas, o reconhecimento de reações adversas potenciais, os problemas que a superdosagem pode ocasionar, pois o que descaracteriza o medicamento como veneno é somente a diferença da dose e as condições adequadas de armazenamento e conservação do produto, desenvolvendo assim uma relação de confiança com o utente, com objetivo de promover o uso ponderado e seguro dos medicamentos e assim garantir a efetividade e segurança terapêutica. Para alcançar maiores resultados, podem ser empregados ainda a prescrição, a atenção farmacêutica e o acompanhamento farmacoterapêutico, para a correção de eventuais erros na farmacoterapia, além da associação à psicoterapia.

\section{REFERÊNCIAS}

ABRACIT. Associação Brasileira de Centros de Informação e Assistência Toxicológica e Toxicologistas Clínicos. Lista de Centros. Brasil, 2012. Disponível em: http://www.abracit.org.br/abracit_site/index.php?option=com_content\&view=artic le\&id=138. Acesso em: 23 mar. 2020.

ARAUJO, E. R. A construção coletiva de uma proposta de planejamento para assistência farmacêutica em uma rede de atenção à saúde. São Carlos, SP. 2016, 89 p. Dissertação (Mestrado) - Universidade Federal de São Carlos, 2016. Disponível em: https://repositorio.ufscar.br/bitstream/handle/ufscar/7398/DissERA.pdf?sequen ce=1\&isAllowed=y. Acesso em: 02 ago. 2020.

ARAUJO, S. Q. et al. Organização dos serviços farmacêuticos no Sistema Único de Saúde em regiões de saúde. Ciênc. saúde coletiva, Rio de Janeiro, v. 22, n. 4, p. 1181-1191, abr. 2017. Disponível em: https://www.scielo.br/pdf/csc/v22n4/1413-8123-csc-22-04-1181.pdf. Acesso em: 01 ago. 2020.

ARAÚJO, S. R.; JUNGES, F. Papel do profissional farmacêutico no âmbito da assistência farmacêutica. In: $8^{\text {a }}$ MOSTRA DE PRODUÇÃO CIENTÍFICA DA PÓS-GRADUAÇÃO LATO SENSU DA PUC GOIÁS, VIII, 2013, Goiás. Anais eletrônicos, Goiás: PUC Goías, 2013, p. $2271 \quad-\quad 2290$. Disponível em: http://www.cpgls.puc goias.edu.br/8mostra/Artigos/Caderno\%208\%20Mostra.pdf. Acesso em: 29 mar. 2020.

BALEN, E. et al. Interações medicamentosas potenciais entre medicamentos psicotrópicos dispensados. J. bras. psiquiatr., Rio de Janeiro, v. 66, n. 3, p. 172-177, set. 2017. Disponível em: http://www.scielo.br/scielo.php?script=sci_arttext \&pid=S004720852017000300172\&lng=en\&nrm=iso. Acesso em: 19 mai. 2020. 
BIZZO, C. V. N. F. et al. A importância da atuação do profissional farmacêutico na saúde mental. Semioses, v. 12, n. 4, p. 145-162, 2018. Disponível em: https:// revistas.unisuam.edu.br/index.php/semioses/article/view/142/95. Acesso em: 01 ago. 2020.

BRASIL, Lei $\mathbf{n}^{\circ}$ 13.021, de 08 de agosto de 2014. Dispõe sobre o exercício e a físcalização das atividades farmacêuticas. Disponível em: http://www.planalto. gov.br/ccivil_03/_Ato2011-2014/2014/Lei/L13021.htm. Acesso em: 11 abr. 2020

BRASIL, Ministério da Saúde. Secretaria de Vigilância em Saúde. Portaria No 344, de 12 de maio de 1998. 1998a. Aprova o Regulamento Técnico sobre substâncias e medicamentos sujeitos a controle especial. Disponível em: https:// bvsms.saude.gov. br/bvs/saudelegis/svs/1998/prt0344_12_05_1998_rep.html. Acesso em: 26 fev. 2020.

BRASIL, Ministério da Saúde. Agência Nacional de Vigilância Sanitária (ANVISA). Resolução de Diretoria Colegiada - RDC $\mathbf{n}^{0}$ 60, de 17 de dezembro de 2010. Estabelece frases de alerta para princípios ativos e excipientes em bulas e rotulagem de medicamentos. Disponível em: https://bvsms.saude.gov.br/bvs/saude legis/anvisa/2010/res0060_ 17_12_2010.html. Acesso em: 09 mai. 2020.

BRASIL, Ministério da Saúde. Cartilha para a promoção do uso racional de medicamentos / Ministério da Saúde, Secretaria de Ciência, Tecnologia e Insumos Estratégicos, Departamento de Assistência Farmacêutica e Insumos Estratégicos. - Brasília: Ministério da Saúde, 2015. 28 p. Disponível: http://bvsms.saude.gov.br/bvs/ publicacoes/cartilha_promocao_uso_racional_medicamentos.pdf. Acesso em: 14 jun. 2020.

BRASIL, Ministério da Saúde. Conselho Nacional de Saúde. Resolução $\mathbf{N}^{\mathbf{0}}$ 338, de 06 de maio de 2004. Disponível em: https://bvsms.saude.gov.br/bvs/saude legis/ cns/2004/res0338_06_05_2004.html. Acesso em: 21 mai. 2020.

BRASIL, Ministério da Saúde. Diretrizes para estruturação de farmácias no âmbito do Sistema Único de Saúde / Ministério da Saúde, Secretaria de Ciência, Tecnologia e Insumos Estratégicos, Departamento de Assistência Farmacêutica e Insumos Estratégicos. Brasília: Ministério da Saúde, 2009. 44 p. : il. - (Série A. Normas e Manuais Técnicos).

BRASIL, Ministério da Saúde. Gabinete do Ministro. Portaria $\mathbf{n}^{\mathbf{0}}$ 3.916, de 30 de outubro de 1998. 1998b. Aprova a Política Nacional de Medicamentos (PNM). Disponível em: https://bvsms.saude. gov.br/bvs/saudelegis/gm/1998/prt3916_30_10_1998.html. Acesso em: 18 mai. 2020.

CARLINI, E. A. et al. Drogas psicotrópicas: o que são e como agem. Revista Imesc, v. 3, p. 9-35, 2001. Disponível em: https://pdfs.semanticscholar.org/8558/9fb446fadb c7cc2ba67e60d2a7e3cf2f1d24.pdf. Acesso em: 12 abr. 2020.

CARVALHO, I. L. N. et al. A intoxicação por psicofármacos com motivação suicida: uma caracterização em idosos. Rev. bras. geriatr. gerontol., Rio de Janeiro, v. 20, n. 1, p. 129137, fev. 2017. Disponível em: http://www.scielo.br/scielo.php?script= sci_arttext\&pid=S1809-98232017000100129\&lng=en\&nrm=iso. Acesso em: 29 fev. 2020.

CARVALHO, I. L. N. Intoxicações por psicofármacos: caracterização, interface com políticas públicas de saúde e tentativa de suicídio. 2018, 101 f. Orientadora: Adriana Rolim

Revista Saúde em Foco, Teresina, v. 7, n. 3, art. 6, p. 76-100, set./dez.2020 www4.fsanet.com.br/revista 
Campos Barros. Tese (Doutorado Acadêmico) - Universidade de Fortaleza. Programa de Saúde Coletiva, Fortaleza, 2018. Disponível em: https:// uol.unifor.br/oul/ObraBdtdSiteTrazer.do?method=trazer \&ns=true\&obra Codigo=105743. Acesso em: 29 mar. 2020.

CETESB, Companhia Ambiental do Estado de São Paulo. Conceitos Básicos em Toxicologia. São Paulo, [21--]. Disponível em: https://cetesb.sp.gov.br/emergenciasquimicas/aspectos-gerais/toxicologia/conceitos-basicos-de-toxicologia/. Acesso em: 13 mar. 2020 .

CONSELHO FEDERAL DE FARMÁCIA. Resolução no 586 de 29 de agosto de 2013. Regula a prescrição farmacêutica e dá outras providências. Disponível em: https://www.cff.org.br/userfiles/file/resolucoes/586.pdf. Acesso em: 07 set. 2020.

CONSTANTINO, V. M. et al. Estoque e descarte de medicamentos no domicílio: uma revisão sistemática. Ciência \& Saúde Coletiva [online]. v. 25, n. 2 , p. 585-594, fev. 2020. Disponível em: https://www.scielosp.org/pdf/csc/2020.v25 n2/585-594/pt. Acesso em: 02 jun. 2020.

CORADI, A. E. P. A importância do farmacêutico no ciclo da Assistência Farmacêutica. Arquivos Brasileiros de Ciências da Saúde, v. 37, n. 2, 2012. Disponível em: http://files.bvs.br/upload/S/1983-2451/2012/v37n2/a3051.pdf. Acesso em: 30 mai. 2020.

COSTA, A. T. Saúde mental: o cuidado farmacêutico inserido em um centro de atenção psicossocial. Experiências Exitosas de Farmacêuticos no SUS, v. 6, n. 6, p. 34-43, 2019. Disponível em: https://cff.org.br/userfiles/Experi\%C3\%AAn cias\% 20Exitosas\%202019.pdf Acesso em: 15 jul. 2020.

COUTO, F. R. et al. Medicamentos psicotrópicos: uso, prescrição e controle. Revista Goiana de Medicina. Goiânia (GO), v.47, ed. 1, p.22-26, 2015. Disponível em: https://repositorio.bc.ufg.br/bitstream/ri/18462/5/Artigo\%20-\%20Fernanda\%20

Rocha\%20Couto\%20-\%202015.pdf. Acesso em: 23 mar. 2020.

DA SILVA, E. R.; ÁLVARES, A. C. M. Intoxicação medicamentosa relacionada à tentativa de autoextermínio. Revista de Iniciação Científica e Extensão, v. 2, n. 2, p. 102-108, fev. 2019. Disponível em: https://revistasfacesa.senaaires.com.br/ index.php/iniciacaocientifica/article/view/154/109. Acesso em: 30 mai. 2020.

FERRARI, C. K. B. et al. Falhas na prescrição e dispensação de medicamentos psicotrópicos: um problema de saúde pública. Journal of Basic and Applied Pharmaceutical Sciences, v. 34, n. 1, 2013. Disponível em: http://rcfba.fcfar. unesp.br/index.php/ojs/article/view/244/242. Acesso em: 08 mai. 2020.

FERREIRA, A. C. Z. et al . A vivência do portador de transtorno mental no uso de psicofármacos na perspectiva do pensamento complexo. Texto contexto - enferm., Florianópolis , v. 26, n. 3, e1000016, 2017. Disponível em: http://www. scielo.br/scielo.php?script=sci_arttext\&pid=S0104-07072017000300306\&lng=em \&nrm=iso. Acesso em: 30 mai. 2020. 
FERREIRA, V. L.; MELO, M. L. S. A importância do seguimento farmacoterapêutico na saúde: uma revisão da literatura. Visão Acadêmica, [S.l.], v. 17, n. 1, p. 125-138, ago. 2016. ISSN 1518-8361. Disponível em: https://revistas. ufpr.br/academica/ article/view/45840/28858. Acesso em: 23 mai. 2020.

FILARDI, A. F. R. O significado do uso dos medicamentos Psicotrópicos na vida cotidiana: Uma revisão sistemática. 2015, 66 f. Orientadora: Djenane Ramalho de Oliveira. Dissertação (mestrado) - Universidade Federal de Minas Gerais, Faculdade de Farmácia, Programa de Pós-Graduação em Medicamentos e Assistência Farmacêutica, Belo Horizonte, 2015. Disponível em: https://repositorio. ufmg.br/bitstream/1843/BUOSAT3NRL/1/filardi_agnes_o_significado_do_uso__ dos_medicamentos_psicotr_picos.pdf. Acesso em: 23 mar. 2020.

FILARDI, A. F. R.; MENDONÇA, S. A. M.; RAMALHO-DE-OLIVEIRA, D. The use of psychotropic medications and non-pharmacological approaches in everyday life: a qualitative study of the lived experience, Rev. latinoam. psicopatol. fundam., São Paulo, v. 22, n. 4, p. 859-883, dez. 2019. Disponível em: https://www.scielo.br/ scielo.php?script=sci_arttext\&pid=S1415-47142019000400859. Acesso em: 30 set. 2020.

FRANCO, A. S.; KRIEGER, J. E. Introdução ao sistema nervoso central. Manual de Farmacologia, Barueri, SP: Ed. Manole, pg. 87-96, 2016.

GONÇALVES, C. A. et al. Intoxicação medicamentosa: relacionada ao uso indiscriminado de medicamentos. Revista Científica da Faculdade de Educação e Meio Ambiente, v. 8, n. 1, p. 135-143, jul. 2017. Disponível em: http://www.faema. edu.br/revistas/index.php/RevistaFAEMA/article/view/449/442. Acesso em: 08 mai. de 2020.

GRETZLER, V. S. et al. Atuação do farmacêutico no URM e na prevenção de intoxicação medicamentosa. Revista Científica da Faculdade de Educação e Meio Ambiente, v. 9, n. edesp, p. 547-550, 15 jun. 2018. Disponível em: http://www. faema.edu.br/revistas/index.php/Revista-FAEMA/article/view/rcf.v9iedesp.580/545. Acesso em: 06 jun. 2020.

LAGO, D. F.; ARGOLO, A. F. L. T. O farmacêutico na dispensação de medicamentos. Revista Científica da Escola Estadual de Saúde Pública de Goiás "Cândido Santiago", v. 5, n. 2, p. 51-63, 2019. Disponível em: http://www. revista.esap.go.gov.br/index.php/resap/article/view/120/157. Acesso em: 21 jul. 2020.

MARQUES, V. B. B. Toxicocinética. In: DORTA, D. J. et al. Toxicologia Forense. São Paulo: Ed. Blucher, pg. 55-64, 2018.

NOGUEIRA, M. J. Capítulo I - Ações gerais dos psicofármacos. In: NOGUEIRA, M. J. O uso de psicofármacos: um guia. 2. ed. Rio de Janeiro: Ed. Atheneu, pg. 95-105, 2017.

NUNES, C. R. M. et al. Panoramas das intoxicações por medicamentos no Brasil. Revista ECiência, v. 5, n. 2, 2017. Disponível em: http://www.revistafjn.com.br/ revista/index.php/eciencia/article/view/247/pdf_247. Acesso em: 07 abr. 2020. 
OLSON, K. R., Seção I - avaliação global e tratamento: diagnóstico de intoxicação e descontaminação, Manual de Toxicologia Clínica. 6. ed. - Dados eletrônicos. - Porto Alegre: AMGH, pg 27-50, 2014.

OMS, Organização Mundial da Saúde. A importância da Farmacovigilância (Monitorização da segurança dos medicamentos), Brasília: OPAS, Organização PanAmericana da Saúde, p. 48, 2005. Disponível em: https://www.paho.org/bra/ index.php?option=com_docman \&view=download\&alias=745-a-importancia-dafarmacovigilancia-5\&category_slug=medicamentos-tecnologia-e-pesquisa-075\& Itemid=965. Acesso em: 30 mai. 2020.

PANDE, M. N. R., AMARANTE, P. D. C, BAPTISTA, T. W. F. Este ilustre desconhecido: considerações sobre a prescrição de psicotrópicos na primeira infância. Ciênc. saúde coletiva, Rio de Janeiro, v. 25, n. 6, p. 2305-2314, jun. 2020. Disponível em: https://www.scielo.br/pdf/ csc/v25n6/1413-8123-csc-25-06-2305.pdf. Acesso em: 30 mai. 2020.

PARANÁ. Secretaria da Saúde. Conceitos Básicos de Toxicologia. Paraná, [21--]. Disponível em: http://www.saude.pr.gov.br/arquivos/File/zoonoses_intoxicacoes/ Conceitos _Basicos_de_Toxicologia.pdf. Acesso em: 10 abr. 2020.

PEREIRA, L. C., FRANCO-BERNARDES, M. F., DORTA, D. J. Fundamentos em Toxicodinâmica. In: DORTA, D. J. et al. Toxicologia Forense. São Paulo: Ed. Blucher, pg. 79-81, 2018.

ROCHA, B. S.; WERLANG, M. C. Psicofármacos na Estratégia Saúde da Família: perfil de utilização, acesso e estratégias para a promoção do uso racional. Ciênc. saúde coletiva, Rio de Janeiro, v. 18, n. 11, p. 3291-3300, nov. 2013. Disponível em: http://www.scielo.br/scielo.php?script= $\quad$ sci_arttext\&pid=S1413-81232013001100 019\&lng=pt\&nrm=iso. Acesso em: 11 jul. 2020.

ROSA, B. P. G. D.; WINOGRAD, M. Sobre o mal-estar hoje: do cérebro à pulsão de morte. Rev. Mal-Estar Subj., Fortaleza, v. 13, n. 1-2, p. 46-80, jun. 2013. Disponível em: http://pepsic.bvsalud.org/scielo.php?script=sci_arttext\&pid=S1518-6148201300 0100003\&lng=pt\&nrm=iso. Acesso em: 8 mar. 2020.

SANTANA, K. S. et al. O papel do profissional farmacêutico na promoção da saúde e do uso racional de medicamentos. Revista Científica da Faculdade de Educação e Meio Ambiente, v. 9, n. 1, p. 399-412, 2018. Disponível em: http://www.faema. edu.br/revistas/index.php/Revista-FAEMA/article/view/538/493. Acesso em: 12 mai. 2020.

SANTOS, L. P.; OLIVEIRA, A. A; SALVI, J. O. Farmacovigilância de medicamentos psicotrópicos no município do Vale do Paraíso, Rondônia. Revista Científica da Faculdade de Educação e Meio Ambiente, v. 6, n. 2, p. 36-48, 16 dez. 2015. Disponível em: http://www.faema.edu.br/revistas/index.php/Revista-FAEMA/ article/view/330/395. Acesso em: 15 abr. 2020.

SANTOS, S. A.; LEGAY, L. F.; LOVISI, G. M. Substâncias tóxicas e tentativas e suicídios: considerações sobre acesso e medidas restritivas. Cad. saúde colet., Rio de Janeiro, v. 21, n. 1, p. 53-61, mar. 2013. Disponível em: http://www.scielo.br/ 
scielo.php?script=sci_arttext\&pid=S1414-462X2013000100009\&lng=en\&nrm=iso. Acesso em: 27 fev. 2020.

SÃO PAULO (Cidade). Secretaria Municipal da Saúde - Prefeitura de São Paulo. Capítulos I - Atendimento inicial das intoxicações agudas e II - O diagnóstico laboratorial toxicológico na suspeita de intoxicação aguda, Manual de Toxicologia Clínica - Orientações para assistência e vigilância das intoxicações agudas. São Paulo, ed.1, p. 9-25, 2017. Disponível em: http://www.cvs.saude.sp.gov.br/up/ MANUAL\%20DE\%20TOXICOLOGIA\%20CL\%C3\%8DNICA\%20-\%20COVISA\% 202017.pdf. Acesso em: 12 abr. 2020.

SARTOR, V. B.; FREITAS, S. F. T. Modelo para avaliação do serviço de dispensação de medicamentos na atenção básica à saúde. Revista de Saúde Pública, v. 48, p. 827-836, 2014. Disponível em: https://www.scielosp.org/ pdf/rsp/2014.v48n5/827-836/pt. Acesso em: 15 ago. 2020.

SILVA, J. C.; HERZOG, L. M. Psicofármacos e psicoterapia com idosos. Psicol. Soc., Belo Horizonte, v. 27, n. 2, p. 438-448, ago. 2015. Disponível em: http:// www.scielo.br/scielo.php?script=sci_arttext\&pid=S0102-71822015000 200438 \&lng=en\&nrm=iso. Acesso em: 19 jul. 2020.

SILVA, O. R. T.; SILVEIRA, M. M. O uso de psicofármacos por crianças e adolescentes em um centro de atenção psicossocial infantil. Infarma - Ciências Farmacêuticas, [S.l.], v. 31, n. 3 , p. 210-218, out. 2019. ISSN 2318-9312. Disponível em: http://revistas.cff.org.br/?journal=infarma\&page $=$ article\&op=view\&path\%5B $\% 5 \mathrm{D} \quad=2527$. Acesso em: 18 abr. 2020.

SILVA, S. N.; LIMA, M. G. Assistência Farmacêutica na Saúde Mental: um diagnóstico dos Centros de Atenção Psicossocial. Ciênc. saúde coletiva, Rio de Janeiro, v. 22, n. 6, p. 20252036, jun. 2017. Disponível em: https://www.scielo.br/ pdf/csc/v22n6/1413-8123-csc-22-062025.pdf. Acesso em: 01 ago. 2020.

SINITOX. Sistema Nacional de Informações Tóxico-Farmacológicas. Casos Registrados de Intoxicação Humana por Agente Tóxico, Sexo e Faixa Etária. Brasil, 2015 à 2017. Rio de Janeiro: Fundação Oswaldo Cruz. FIOCRUZ. 2020. Disponível em: https://sinitox.icict.fiocruz.br/dados-nacionais. Acesso em: 23 mar. 2020.

VIERA, B. Estudantes de Medicina e o uso indiscriminado de ritalina: drogas da inteligência, saúde mental, suicídio, FMUSP - Revista TRIP, São Paulo, jun. 2017. Disponível em: https://revistatrip.uol.com.br/trip/estudantes-de-medicina-e-o-uso-indiscriminado-de-ritalinadrogas-da-inteligencia-saude-mental-suicidio-fmusp\#. Acesso em: 17 mai. 2020

ZANELlA, C. G.; AGUIAR, P. M.; STORPIRTIS, S. Atuação do farmacêutico na dispensação de medicamentos em Centros de Atenção Psicossocial Adulto no município de São Paulo, SP, Brasil. Ciênc. saúde coletiva, Rio de Janeiro, v. 20, n. 2, p. 325-332, fev. 2015. Disponível em: http://www.scielo.br/scielo.php?script =sci_arttext\&pid=S1413$81232015000200325 \& \operatorname{lng}=e n \& n r m=i s o$. Acesso em: 20 jul. 2020. 


\section{Como Referenciar este Artigo, conforme ABNT:}

CSHUNDERLICK, C; ZAMBERLAM, C. R. Análise das Principais Intervenções Fisioterapêuticas Usadas em Pacientes Vítimas de Acidente Vascular Cerebral. Rev. Saúde em Foco, Teresina, v. 7, n. 3, art. 6, p. 76-100, set./dez.2020.

\begin{tabular}{|l|c|c|}
\hline \multicolumn{1}{|c|}{ Contribuição dos Autores } & C. Cshunderlick & $\begin{array}{c}\text { C. } \\
\text { Zamberlam }\end{array}$ \\
\hline 1) concepção e planejamento. & $\mathrm{X}$ & $\mathrm{X}$ \\
\hline 2) análise e interpretação dos dados. & $\mathrm{X}$ & $\mathrm{X}$ \\
\hline 3) elaboração do rascunho ou na revisão crítica do conteúdo. & $\mathrm{X}$ & $\mathrm{X}$ \\
\hline 4) participação na aprovação da versão final do manuscrito. & $\mathrm{X}$ & $\mathrm{X}$ \\
\hline
\end{tabular}

